Research article

Open Access

\title{
Characterization of tamoxifen and 4-hydroxytamoxifen glucuronidation by human UGT1A4 variants
}

\author{
Dongxiao Sun ${ }^{1}$, Gang Chen ${ }^{2}$, Ryan W Dellinger ${ }^{1}$, Kimberly Duncan ${ }^{1}$, Jia-Long Fang ${ }^{1,3}$ and \\ Philip Lazarus ${ }^{1,2}$
}

\begin{abstract}
${ }^{1}$ Cancer Prevention and Control Program, Division of Population Sciences and Cancer Prevention, Department of Pharmacology, Penn State Cancer Institute, Penn State University College of Medicine, Hershey, Pennsylvania, USA

${ }^{2}$ Cancer Prevention and Control Program, Division of Population Sciences and Cancer Prevention, Department of Health Evaluation Sciences, Penn State Cancer Institute, Penn State University College of Medicine, Hershey, Pennsylvania, USA

${ }^{3}$ Division of Biochemical Toxicology, National Center for Toxicological Research, Jefferson, Arkansas, USA
\end{abstract}

Corresponding author: Philip Lazarus, plazarus@psu.edu

Received: 20 Apr 2006 Revisions requested: 7 Jul 2006 Revisions received: 28 Jul 2006 Accepted: 2 Aug 2006 Published: 2 Aug 2006

Breast Cancer Research 2006, 8:R50 (doi:10.1186/bcr1539)

This article is online at: http://breast-cancer-research.com/content/8/4/R50

(C) 2006 Sun et al., licensee BioMed Central Ltd.

This is an open access article distributed under the terms of the Creative Commons Attribution License (http://creativecommons.org/licenses/by/2.0), which permits unrestricted use, distribution, and reproduction in any medium, provided the original work is properly cited.

\begin{abstract}
Introduction Tamoxifen (TAM) is an antiestrogen widely used in the treatment and prevention of breast cancer in women. One of the major mechanisms of metabolism of TAM and one of its major active metabolites, 4-hydroxytamoxifen (4-OH-TAM), is via glucuronidation. In the present study, the glucuronidating activities of three common variant isoforms encoded by the human UDP-glucuronosyltransferase (UGT) 1A4 gene were examined against TAM, trans-4-OH-TAM and cis-4-OH-TAM.
\end{abstract}

Methods HPLC was used to detect glucuronide conjugates in microsomes from UGT1A4-overexpressing HK293 cells. The UGT1A4 wild-type cDNA was synthesized by RT-PCR using normal human liver total RNA. The UGT1A424Thr/48Leu and UGT1A424Pro/48Val variants were generated by site-directed mutagenesis of the pcDNA3.1/V5-His-TOPO plasmid expressing wild-type UGT1A424Pro/48Leu. Levels of UGT1A4 expression in UGT-overexpressing cell lines were measured by western blot analysis.

Results Microsomes from wild-type UGT1A424Pro/48Leu overexpressing HK293 cells exhibited significant levels of activity against TAM, trans-4-OH-TAM and cis-4-OH-TAM, forming exclusively the tamoxifen quaternary ammonium glucuronide (TAM- $N^{+}$-glucuronide) and the 4-hydroxytamoxifen quaternary ammonium glucuronides (trans-4-OH-TAM- $N^{+}$- glucuronide and cis-4-OH-TAM- $N^{+}$-glucuronide) with apparent $K_{\mathrm{m}}$ values of $2.0 \mu \mathrm{M}, 2.2 \mu \mathrm{M}$, and $2.1 \mu \mathrm{M}$, respectively. Higher glucuronidation activities were found by kinetic analysis for microsomes from the variant UGT1A424Pro/48Val-overexpressing cell line as compared with microsomes from wild-type UGT1A424Pro/48Leu-overexpressing cells against TAM and against both the trans and cis isomers of 4-OH-TAM. A significantly $(P<0.02)$ lower $K_{\mathrm{m}}$ value $(\sim 1.6$-fold to 1.8 -fold $)$ was observed for both 4-OH-TAM isomers, while a nearsignificant $(P=0.053)$ decrease in $K_{\mathrm{m}}$ was observed for TAM for the UGT1A424Pro/48Val variant as compared with wild-type UGT1A4. The $V_{\max } / K_{\mathrm{m}}$ ratio for the UGT1A424Pro/48Val variant was significantly $(P \leq 0.005)$ higher than that observed for the wild-type UGT1A4 isoform for both the trans and cis isomers of 4-OH-TAM after normalization for UGT1A4 expression by western blotting. No significant effect on enzyme kinetics was observed for the UGT1 A424Thr/48Leu variant against either isomer of $4-\mathrm{OH}-\mathrm{TAM}$ or with TAM.

Conclusion These data suggest that the UGT1A4 codon 48 Leu $>$ Val polymorphism significantly alters glucuronidation rates against TAM and its active hydroxylated metabolites, and that this polymorphism may play an important role in individual pharmacological response to TAM therapy.

$\overline{\mathrm{DMEM}}=$ Dulbecco's modified Eagle's medium; HPLC = high-performance liquid chromatography; $K_{\mathrm{m}}=$ Michaelis-Menten equilibrium constant; LCMS = liquid chromatography-mass spectrometry; Leu = leucine; 4-OH-TAM = 4-hydroxytamoxifen; 4-OH-TAM- $N^{+}$-glucuronide = 4-hydroxytamoxifen quaternary ammonium glucuronide; TAM = tamoxifen; TAM- $N^{+-}$-glucuronide = tamoxifen quaternary ammonium glucuronide; $P C R=$ polymerase chain reaction; Pro = proline; RT = reverse transcriptase; Thr = threonine; UDPGA = UDP-glucuronic acid; UGT = UDP-glucuronosyltransferase; Val = valine. 


\section{Introduction}

Tamoxifen (TAM) (1- [4-(2-dimethylaminoethoxy)-phenyl]-1,2diphenylbut-1(Z)-ene) is a nonsteroidal antiestrogen commonly used for the treatment and prevention of estrogendependent breast cancer [1-4]. Adjuvant TAM treatment increases recurrence-free survival and overall survival in breast cancer patients with hormone receptor-positive tumors irrespective of their nodal status, menopausal status or age [5]. In addition to its antiestrogenic properties, which have been related to symptoms such as hot flashes, vaginal bleeding and pruritus vulvae [2,6], TAM also has partial estrogen-agonistic effects that may be linked to reduced risk for ischemic heart disease and osteoporosis [7,8], but may also increase the risk for endometrial cancer $[9,10]$ and venous thromboembolism [11]. Although TAM is generally well tolerated, there is significant interindividual variability in the clinical efficacy of TAM as well as in the toxicities of TAM. For instance, about $30 \%$ of patients acquire TAM resistance and relapse [12]. In addition, the relative risk of endometrial cancers in patients treated with TAM is estimated to be twofold to threefold that of controls, the risk increasing with both the duration of and cumulative dose of TAM treatment [10,13-15].

The mechanisms underlying variability in response to TAM and to TAM-related toxicities remain obscure. Since there is compelling evidence that TAM is converted to antiestrogenic metabolites more potent than TAM itself, one hypothesis is that altered patterns of metabolism of TAM and/or its primary metabolites might contribute to this interindividual variability.

TAM is activated predominantly via cytochrome P450-mediated pathways into several metabolites after oral administration, including the hydroxylated TAM metabolites, 4hydroxytamoxifen (4-OH-TAM) and 4-hydroxy- $N$-desmethylTAM (endoxifen). Since both trans-4-OH-TAM and endoxifen exhibit up to 100 -fold the levels of antiestrogenic activity compared with TAM and other TAM metabolites [16-21], it is thought they may be the major contributors to TAM's antiestrogenic properties. While cis-4-OH-TAM is thought to be primarily an estrogen agonist, this isomer exhibits significant antiestrogenic activity in vitro when in the presence of estradiol [22-24].

An important route of elimination and detoxification of TAM and its metabolites is via glucuronidation. TAM is excreted predominantly through the bile, a process largely facilitated by TAM conjugation to glucuronic acid during the glucuronidation process [25], and TAM glucuronides have been identified in the urine of TAM-treated patients [26]. Most of the 4-OH-TAM in the bile of TAM-treated patients was found as a glucuronide conjugate $[25,27]$. The fact that TAM metabolites are found in their unconjugated form in feces is probably due to $\beta$-glucuronidase-catalyzed deglucuronidation within the microflora that colonize within the small intestine [25]. TAM glucuronide conjugates have been identified in the serum of TAM-treated patients [25,27], and it has been suggested that glucuronidation within target tissues such as the adipose tissue of the breast may also be important in terms of TAM metabolism and overall TAM activity [28].

One of the major UDP-glucuronosyltransferases (UGTs) involved in the glucuronidation of TAM and its metabolites is the hepatic enzyme UGT1A4 [29,30], which catalyzes the formation of a quarternary ammonium-linked glucuronide with TAM's N, N-dimethylaminoalkyl side chain [30]. This pattern of ammonium-linked glucuronidation is consistent with UGT1A4's glucuronidation activity against primary, secondary and tertiary amines present in a variety of carcinogenic compounds, androgens, progestins and plant steroids [31-34].

Two unlinked missense polymorphisms were identified at codon 24 (Pro>Thr) and codon 48 (Leu>Val) within the UGT1A4 gene $[35,36]$. The prevalence of both variant UGT1A424Thr/48Leu and UGT1A424Pro/48Val alleles approached 0.10 in Caucasian populations [35,36]. The codon 24 polymorphism was linked to altered glucuronidation activity against the tobacco-specific nitrosamine, 4(methylnitrosamino) 1 (3pyridyl) 1 butanol [36], while the UGT1A448Val variant was associated with decreased rates of glucuronidation after transient transfection into cell lines in vitro [35].

The aim of the present study was to characterize UGT1A4induced glucuronidation of TAM, trans-4-OH-TAM and cis-4$\mathrm{OH}-\mathrm{TAM}$, and to examine whether missense variants in the UGT1A4 gene alter activity against TAM and its hydroxylated metabolites in vitro.

\section{Materials and methods Chemicals and materials}

UDP-glucuronic acid (UDPGA), trans-TAM, trans-4-OH-TAM (98\% pure), trans-4-OH-TAM:cis-4-OH-TAM (70:30\% ratio), alamethicin, $\beta$-glucuronidase, anticalnexin antibody and bovine serum albumin were purchased from Sigma-Aldrich (St Louis, MO, USA). HPLC-grade ammonium acetate and acetonitrile were purchased from Fisher Scientific (Pittsburgh, PA, USA) and were used after filtration. DMEM, Dulbecco's phosphatebuffered saline (minus calcium chloride and magnesium chloride), fetal bovine serum, penicillin-streptomycin and geneticin (G418) were purchased from Gibco (Grand Island, NY, USA).

The Platinum ${ }^{\circledR} P f x$ DNA polymerase and the pcDNA3.1/V5His-TOPO mammalian expression vector were obtained from Invitrogen (Carlsbad, CA, USA), while the restriction enzymes Dpn/ and Stu/ were purchased from New England Biolabs (Beverly, MA, USA). The BCA protein assay kit was purchased from Pierce (Rockford, IL, USA) while the QIAEX ${ }^{\circledR}$ II gel extraction kit was purchased from Qiagen (Valencia, CA, USA). The human UGT1A western blotting kit and anti-UGT1A antibody were purchased from Gentest (Woburn, MA, USA). All other 
chemicals used were purchased from Fisher Scientific (Pittsburgh, PA, USA) unless otherwise specified.

\section{Tissues}

A description of the normal human liver tissue specimens used for these studies was presented previously [36]. Briefly, tissues were quick-frozen at $-70^{\circ} \mathrm{C}$ within 2 hours postsurgery. Liver microsomes were prepared through differential centrifugation as previously described [37] and were stored (10-20 $\mathrm{mg}$ protein $/ \mathrm{ml}$ ) at $-70^{\circ} \mathrm{C}$. Microsomal protein concentrations were measured using the BCA assay (Pierce). All protocols involving the analysis of tissue specimens were approved by the institutional review board at the Penn State College of Medicine and in accordance with assurances filed with and approved by the United States Department of Health and Human Services.

\section{UGT1A4 cloning}

The UGT1A4 wild-type cDNA was synthesized by RT-PCR using normal human liver total RNA and was inserted into the pcDNA3.1/V5-His-TOPO plasmid. The sense and antisense primers used for RT-PCR were UGT1A4s1 (5'-ACAGTCAGCTGTCGGTGGC-3', corresponding to -29 to -11 relative to the UGT1A4 translation start site; GenBank accession number NM007120) and UGT1A4as1 (5'-ATTTTACCTTATTTCCCACCC-3', corresponding to +1611 to +1631 relative to the UGT1A4 translation start site), respectively.

Incubations were performed in a GeneAmp 9700 thermocycler (Applied Biosystems, Foster City, CA, USA) as follows: one cycle at $94^{\circ} \mathrm{C}$ for 2 minutes, 41 cycles at $94^{\circ} \mathrm{C}$ for 30 seconds, at $55^{\circ} \mathrm{C}$ for 30 seconds and at $72^{\circ} \mathrm{C}$ for 2 minutes, followed by a final cycle of 7 minutes at $72^{\circ} \mathrm{C}$. The PCR product (1662 base pairs) was purified after electrophoresis in 1.5\% agarose using the QIAEX ${ }^{\circledR}$ || gel extraction kit (Qiagen), and was subsequently subcloned into the pcDNA3.1/V5-HisTOPO mammalian expression vector using standard methodologies.

Confirmation of insert orientation was performed by restriction enzyme digestion, and UGT1A4 sequences were confirmed by dideoxy sequencing of the entire PCR-amplified UGT1A4 cDNA product (performed at the Molecular Biology Core Facility at Penn State University College of Medicine) using two vector primers (T7 and BGH; IDT, Coralville, IA, USA) and one internal sense primer (UGT1A4s2 - 5'-GAAGGAATTTGATCGCGTTAC-3', corresponding to nucleotides +258 to +278 relative to the UGT1A4 translation start site). The cloned UGT1A4 insert was compared with that described in GenBank and was confirmed to be $100 \%$ homologous to the wild-type UGT1A424Pro/48Leu sequence (accession number NM007120).

\section{Generation of UGT1A4 variants, overexpressing cell lines and cell microsome preparation}

The UGT1A424Thr/48Leu and UGT1A424Pro/48Val variants were generated by site-directed mutagenesis of the pcDNA3.1/V5His-TOPO plasmid expressing wild-type UGT1A424Pro/48Leu. The primers used to change codon 24 from Pro to Thr were UGT1A4c24F (5'-CAGTGTCCAGACCTGGGCTGAGAGTG-3') and UGT1A4c24R (5'-CACTCTCAGCCCAGGTCTGGACACTG-3'), both primers corresponding to nucleotides +60 to +85 relative to the UGT1A4 translation start site (mutated base indicated in bold). The primers used to change codon 48 from Leu to Val were UGT1A4c48F (5'CTCAGCATGCGGGAGGCCGTGCGGGAGCTCCATGC$\left.3^{\prime}\right)$ and UGT1A4c48R (5'-GCATGGAGCTCCCGCACGGCCTCCCGCATGCTGAG-3'), both primers corresponding to nucleotides +124 to +158 relative to the UGT1A4 translation start site.

The products were amplified by PCR using $5 \mathrm{U} / \mathrm{ml} P f x$ polymerase, $1 \times$ Pfx buffer, $2 \times$ enhancer, $10 \mu \mathrm{M}$ dNTPs, 500 ng template, $1 \mathrm{mM} \mathrm{MgSO}_{4}$ and $20 \mu \mathrm{M}$ of each primer in a BioRad MyCycler (Hercules, CA, USA) with an initial denaturation at $95^{\circ} \mathrm{C}$ for 2 minutes, followed by 25 cycles at $95^{\circ} \mathrm{C}$ for 30 seconds, at $65^{\circ} \mathrm{C}$ for 30 seconds and at $68^{\circ} \mathrm{C}$ for 18 minutes. Following amplification, $20 \mathrm{U}$ Dpn Irestriction enzyme was added to each reaction and incubated for 1 hour at $37^{\circ} \mathrm{C}$ to specifically digest the wild-type template DNA. The remaining $\mathrm{PCR}$ product was then transformed into competent $\mathrm{DH} 5 \alpha$ Escherichia coli, individual colonies were isolated, and subsequent plasmid DNA minipreps were screened for the UGT1A424Thr/48Leu and UGT1A424Pro/48Val variants using HPY188III or Stul, respectively. The UGT1A424Thr/48Leu and UGT1 A424Pro/48Val CDNA sequences were confirmed by direct dideoxy sequencing using the same primers used for wild-type UGT1A4 analysis described earlier.

Human embryonic kidney fibroblast HEK293 cell lines overexpressing wild-type or variant UGT1A4 were generated by standard electroporation techniques in the Bio-Rad GenePulser Xcell using $10 \mu \mathrm{g}$ pcDNA3.1/V5-His-TOPO/ UGT1A4 plasmid DNA with $5 \times 10^{6} \mathrm{HEK} 293$ cells (in $0.5 \mathrm{ml}$ ) in serum-free DMEM media, with electroporation at $250 \mathrm{~V}$ and $1000 \mu \mathrm{F}$. Following transfection, HK293 cells were grown in $5 \% \mathrm{CO}_{2}$ to $80 \%$ confluence in DMEM supplemented with 4.5 $\mathrm{mM}$ glucose, $10 \mathrm{mM}$ HEPES, $10 \%$ fetal bovine serum, $100 \mathrm{U} /$ $\mathrm{ml}$ penicillin, $100 \mu \mathrm{g} / \mathrm{ml}$ streptomycin and geneticin $(700 \mu \mathrm{g} /$ $\mathrm{ml}$ medium) for the selection of geneticin-resistant clones, with selection medium changed every 3-4 days.

Individual UGT1A4-overexpressing cell colonies were selected and monitored for UGT1A4 expression via western blotting analysis (described in the next section). Cell homogenates were prepared by resuspending pelleted cells in Trisbuffered saline $(25 \mathrm{mM}$ Tris base, $138 \mathrm{mM} \mathrm{NaCl}, 2.7 \mathrm{mM} \mathrm{KCl}$, $\mathrm{pH} 7.4$ ) and subjecting them to three rounds of freeze-thaw 
prior to gentle homogenization. Microsomal fractions were prepared by centrifugation of whole cell homogenate at $10,500 \mathrm{rpm}(9,000 \times \mathrm{g})$ for 30 minutes at $4^{\circ} \mathrm{C}$; the supernatant was collected and subsequently centrifuged at 33,500 rpm $(105,000 \times g)$ for 60 minutes at $4^{\circ} \mathrm{C}$ in a SW-55 Ti rotor (Beckman, Palo Alto, CA, USA). Pellets were collected by suspension in Tris-buffered saline (25 mM Tris base, $138 \mathrm{mM}$ $\mathrm{NaCl}, 2.7 \mathrm{mM} \mathrm{KCl}, \mathrm{pH} 7.4)$ and were stored at $-80^{\circ} \mathrm{C}$ in 100 $\mu$ aliquots. Total microsomal protein concentrations were measured using the BCA protein assay (Pierce).

\section{Western blot analysis}

Levels of UGT1A4 expression in UGT-overexpressing cell lines were measured by western blot analysis using the antiUGT1A antibody in a 1:5,000 dilution as per the manufacturer's instructions (Gentest), while calnexin protein levels were assayed using a 1:5,000 dilution of the monoclonal anticalnexin antibody (after stripping the UGT1A4 antibody of the same western blot using standard techniques). UGT1A4 was detected by chemiluminescence using the SuperSignal West Dura Extended Duration Substrate (Pierce Biotechnology, Inc., Rockford, IL, USA). Secondary antibodies supplied with the Dura ECL kit (anti-rabbit and anti-mouse) were used at 1:3,000. UGT1A4 levels were quantified against a known amount of human UGT1A protein (200-300 ng, supplied in the western blotting kit; Gentest) by densitometric analysis of $X$-ray film exposures (1 s-1 min exposures) of western blots using a GS-800 densitometer with Quantity One software (Bio-Rad).

UGT1A4 protein levels were calculated against known titrated quantities of UGT1A standard, with quantification made relative to the levels of calnexin observed in each lane (quantified by densitometric analysis of western blots as already described). Determinations of aglycone-glucuronide formation in UGT1A4-overexpressing cell lines were calculated relative to the levels of UGT1A4 expression in the respective cell lines. $\mathrm{X}$-ray film bands were always below densitometer saturation levels as indicated by the densitometer software. Densitometric results were always consistent irrespective of the exposure time. Western blot analysis and subsequent densitometric analysis were performed in triplicate on three separate occasions, using the same UGT1A4-containing cell homogenates used for activity assays, with relative UGT1A4 protein levels expressed as the mean of these experiments.

\section{Glucuronidation assays}

Glucuronidation activities of microsomes from human UGT1A4-overexpressing HEK293 cells toward trans-TAM, trans-4-OH-TAM and cis-4-OH-TAM were performed after an initial incubation of microsomal protein $(5-25 \mu \mathrm{g})$ with alamethicin $(50 \mu \mathrm{g} / \mathrm{mg}$ protein) for 15 minutes in an ice bath. Glucuronidation reactions were then performed in a final reaction volume of $100-500 \mu \mathrm{l}$ at $37^{\circ} \mathrm{C}$ in $50 \mathrm{mM}$ Tris- $\mathrm{HCl}(\mathrm{pH} \mathrm{7.4),}$ $10 \mathrm{mM} \mathrm{MgCl}_{2}, 4 \mathrm{mM}$ UDPGA and 1-6 $\mu \mathrm{M}$ trans-TAM or trans-
4-OH-TAM, and 1-15 $\mu \mathrm{M}$ cis-4-OH-TAM. Reactions were terminated by the addition of 95-495 $\mu \mathrm{l}$ cold methanol on ice. Five microliters of propranolol $(2.5 \mu \mathrm{g} / \mathrm{ml})$ was added to the final reactions, the mixtures were centrifuged for 10 minutes at $4^{\circ} \mathrm{C}$ at $16,100 \times g$, the supernatants were collected and evaporated, and the resulting dried sample was resuspended in $200 \mu$ of $50 \%$ methanol. Assays with human liver microsomes were identical except that $10 \mu \mathrm{g}$ microsomal protein was used in glucuronidation assays.

Samples $(100 \mu \mathrm{l})$ were analyzed for glucuronidated TAM or for TAM metabolites by HPLC using a Beckman Coulter System Gold 126 Solvent Module HPLC system (Fullerton, CA, USA) equipped with an automatic injector (model 508) and a UV detector operated at $254 \mathrm{~nm}$ (model 168). HPLC was performed using a $3 \mu$ Luna $_{18}$ analytical column $(4.6 \mathrm{~mm} \times 150$ $\mathrm{mm}$; Phenomenex, Torrance, CA, USA) in series with a $5 \mu$ Aquasil $\mathrm{C}_{18}$ guard column (10 mm $\times 4 \mathrm{~mm}$; Thermo HypersilKeystone, Bellefonte, PA, USA). The gradient elution conditions for assays with trans-4-OH-TAM or cis-4-OH-TAM were as follows: starting with $75 \%$ buffer $\mathrm{A}$ (100 mM ammonium acetate, $\mathrm{pH} 5.0$ ) and $25 \%$ acetonitrile (5 min), a subsequent linear gradient to $75 \%$ acetonitrile (25\% buffer A) over 25 min was performed and then maintained at $75 \%$ acetonitrile for 10 minutes. The flow rate was $0.5 \mathrm{ml} / \mathrm{min}$. For assays with TAM, the linear gradient was from $70 \%$ buffer A (30\% acetonitrile) to $90 \%$ acetonitrile (10\% buffer $A$ ) over 30 minutes.

The amount of $N^{+}$-glucuronide formed was calculated based on the ratio of the peak area of the $N^{+}$-glucuronide versus that observed for the internal standard, propranolol. Tamoxifen quaternary ammonium glucuronide (TAM- $N^{+}$-glucuronide), the 4-hydroxytamoxifen quaternary ammonium glucuronides (trans-4-OH-TAM- $N^{+}$-glucuronide and cis-4-OH-TAM- $N^{+}$-glucuronide), and trans-4-OH-TAM-O-glucuronide and cis-4-OHTAM-O-glucuronide were confirmed by $1 \mathrm{M} \mathrm{NaOH}$ hydrolysis and sensitivity to $\beta$-glucuronidase as previously described [34]. As controls, glucuronidation assays were regularly performed using human liver microsomes (as a positive control for glucuronidation activity) and untransfected HK293 cell homogenate protein (as a negative control for glucuronidation activity) as previously described [34,38]. Experiments were always performed in triplicate in independent assays.

\section{Liquid chromatography-mass spectrometry identification of 4-OH-TAM-N+-glucuronides}

The predicted trans-4-OH-TAM- $N^{+}$-glucuronide and cis-4$\mathrm{OH}$-TAM- $\mathrm{N}^{+}$-glucuronide were collected after separation by HPLC as already described and were identified by liquid chromatography-mass spectrometry (LC-MS) using a Shimadzu LC-MS 2010 EV system (Shimadzu, Tokyo, Japan). The trans4-OH-TAM- $N^{+}$-glucuronide and cis-4-OH-TAM- $N^{+}$-glucuronide were loaded onto a Shimadzu reverse-phase column (Shimadzu C18, $4.6 \mathrm{~mm} \times 50 \mathrm{~mm}$ ) and were analyzed at a flow rate of $0.2 \mathrm{ml} / \mathrm{min}$ by applying a linear mobile phase gra- 

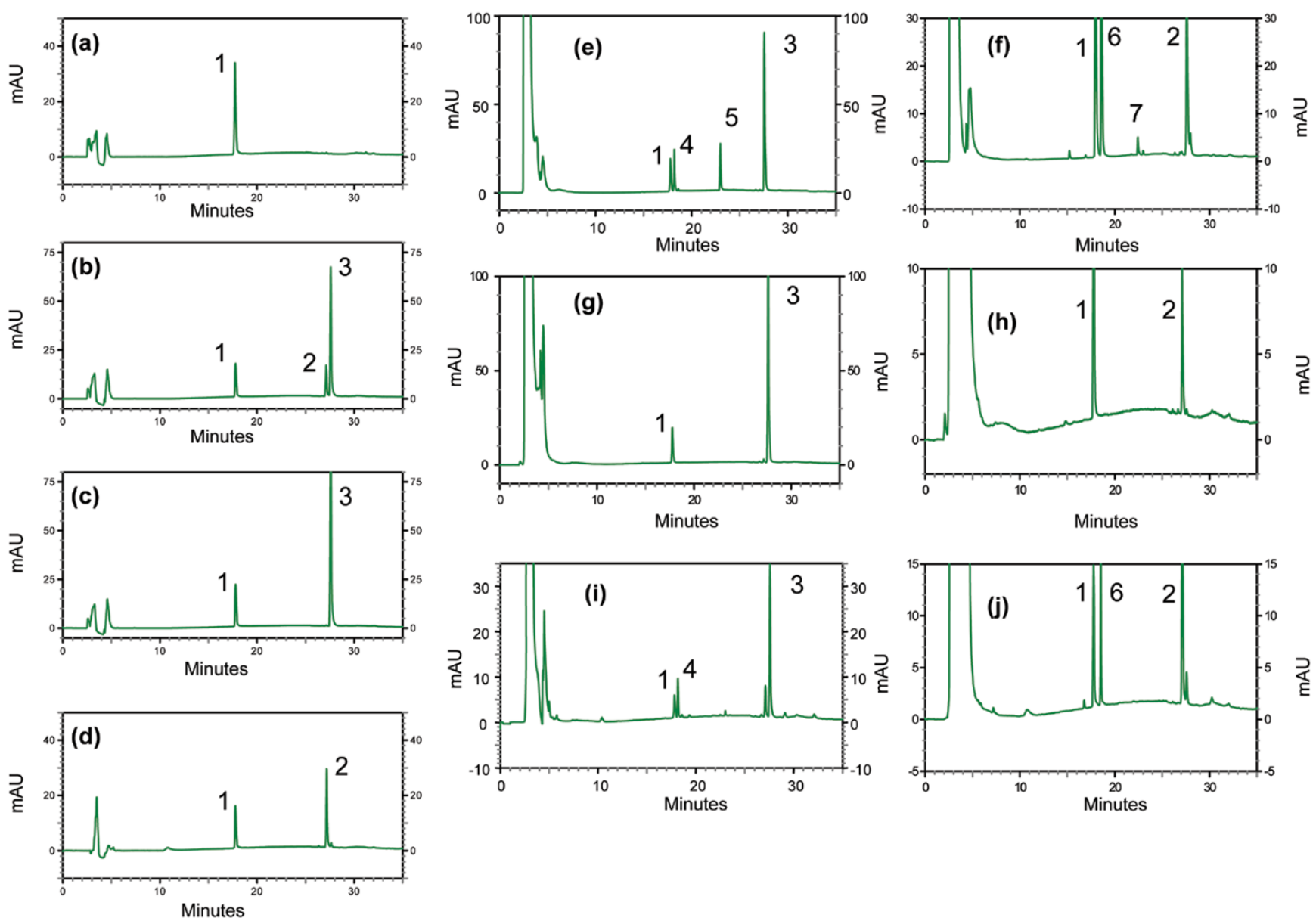

HPLC analysis of 4-hydroxytamoxifen glucuronides formed by human liver microsomes. Human liver microsomes (HLM; $10 \mu \mathrm{g}$ protein) were incubated at $37^{\circ} \mathrm{C}$ for $30 \mathrm{~min}$ with $25 \mu \mathrm{M}$ trans-4-hydroxytamoxifen (trans-4-OH-TAM) or $15 \mu \mathrm{M}$ cis-4-OH-TAM and $4 \mathrm{mM}$ UDP-glucuronic acid prior to analysis by HPLC as described in Materials and methods. Propranolol was added to all assays postincubation as an internal standard for HPLC. (a) No substrate, internal standard (propranolol) only; (b) nonincubated 70:30 mixture of trans-4-OH-TAM:cis-4-OH-TAM (Sigma); (c) nonincubated 98\% pure trans-4-OH-TAM (Sigma); (d) nonincubated cis-4-OH-TAM purified from the 70:30 mixture shown in (b); (e) trans-4-OH-TAM incubated with HLM; (f) cis-4-OH-TAM incubated with HLM; (g) trans-4-OH-TAM incubated with HLM and $\beta$-glucuronidase; (h) cis-4-OH-TAM incubated with $\mathrm{HLM}$ and $\beta$-glucuronidase; (i) trans-4-OH-TAM incubated with HLM and $1 \mathrm{~N} \mathrm{NaOH}$; (j) cis-4-OH-TAM incubated with HLM and $1 \mathrm{~N} \mathrm{NaOH}$. Peak 1 , propranolol; peak 2, cis-4-OH-TAM; peak 3, trans-4-OH-TAM; peak 4, trans-TAM-4-O-glucuronide (predicted); peak 5, trans-4-OH-TAM- $N^{+}$-glucuronide (predicted); peak 6, cis-TAM-4-O-glucuronide (predicted); peak 7, cis-4-OH-TAM- $N^{+}$-glucuronide (predicted). mAU, milli-absorbance units.

dient from $10 \%$ to $80 \%(\mathrm{v} / \mathrm{v})$ methanol/ $\mathrm{H}_{2} \mathrm{O}$ over 30 minutes. An electrospray voltage of $1.5 \mathrm{kV}$ was applied using a positive mode.

\section{Statistical analysis}

The Student $t$ test (two-sided) was used for comparing the rates and the kinetic values of glucuronide formation for the UGT1A424Pro/48Leu, UGT1A424Thr/48Leu and UGT1A424Pro/48Val variant isoforms against the different substrates examined in this study. Kinetic constants were determined using Graphpad Prism4 software (GraphPad Software, San Diego, CA, USA).

\section{Results \\ Characterization of 4-HO-TAM glucuronides in microsomes from human liver and UGT1A4- overexpressing cells}

While $N^{+}$-glucuronide was shown to be the only glucuronide formed with TAM in human liver microsomes and UGT1A4overexpressing baculosomes [30], 4-OH-TAM O-glucuronides and $\mathrm{N}^{+}$-glucuronides were formed by human liver microsomes. As shown in Figure 1, a HPLC assay was developed to efficiently separate TAM and TAM metabolites from their glucuronide conjugates. 


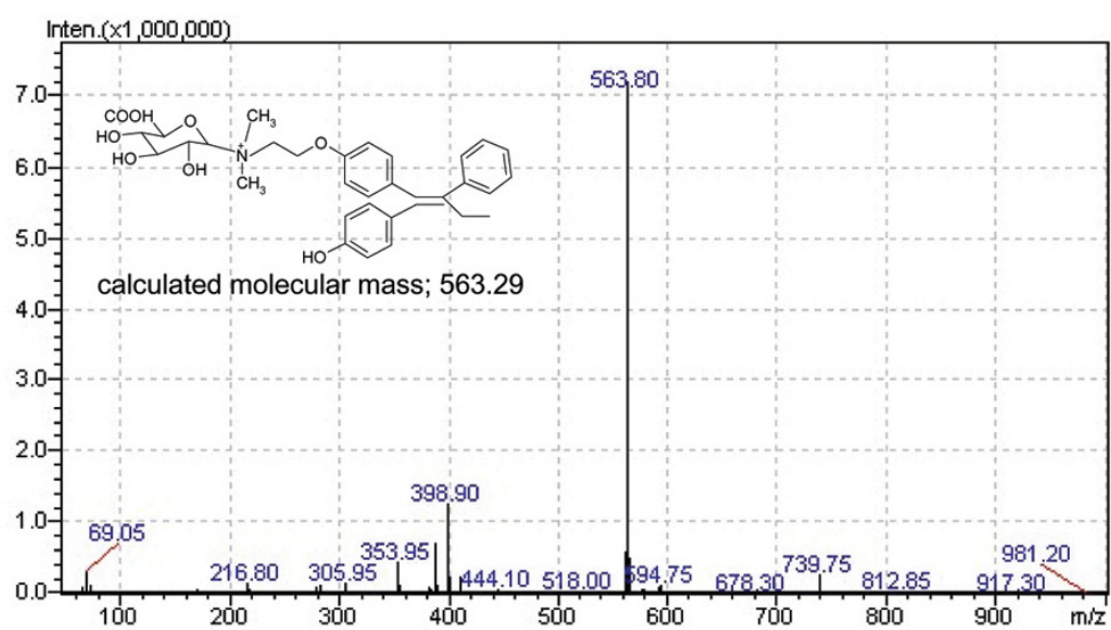

Representative mass spectra of trans-4-hydroxytamoxifen quaternary ammonium glucuronide formed by human liver microsomes. The predicted trans-4-hydroxytamoxifen quaternary ammonium glucuronide (trans-4-OH-TAM- $N^{+}$-glucuronide) was collected after separation by HPLC and identified by liquid chromatography-mass spectrometry using a Shimadzu LC-MS 2010 EV system. trans-4-OH-TAM- $\mathrm{N}^{+}$-glucuronide was loaded onto a C18 Shimadzu reverse-phase column and analyzed at a flow rate of $0.2 \mathrm{ml} / \mathrm{min}$ by applying a linear mobile phase gradient from $10 \%$ to $80 \%$ (v/v) methanol/ $/ \mathrm{H}_{2} \mathrm{O}$ over $30 \mathrm{~min}$. An electrospray voltage of $1.5 \mathrm{kV}$ was applied using a positive mode.

Using propranolol as an internal standard (Figure 1a), the cis and trans isomers of 4-OH-TAM eluted at retention times of 27.1 minutes and 27.6 minutes, respectively, as shown by HPLC of a 70:30 mix of trans-4-OH-TAM:cis-4-OH-TAM (Sigma) (Figure 1b), of 98\% pure trans-4-OH-TAM (Sigma) (Figure 1c), and of cis-4-OH-TAM purified from the 70:30 trans-4-OH-TAM:cis-4-OH-TAM mix (Sigma) (Figure 1d). In assays with human liver microsomes, two 4-OH-TAM glucuronide peaks with retention times of 18.2 minutes (peak 4) and 23.0 minutes (peak 5) were observed in assays with trans-4$\mathrm{OH}-\mathrm{TAM}$ (Figure 1e), and two 4-OH-TAM glucuronide peaks with retention times of 18.6 minutes (peak 6) and 22.5 minutes (peak 7) were observed in assays with cis-4-OH-TAM (Figure 1f). Peaks 4-7 were sensitive to treatment with $\beta$-glucuronidase in glucuronidation assays with either trans-4-OHTAM (Figure 1g) or cis-4-OH-TAM (Figure 1h). Only peaks 5 and 7 were sensitive to treatment with alkali after glucuronidation assays with either trans-4-OH-TAM (Figure 1i) or cis-4$\mathrm{OH}-\mathrm{TAM}$ (Figure 1j).

This pattern is similar to that observed for $N$-glucuronide versus O-glucuronide deconjugation for other compounds [32] suggesting that peaks 4 and 6 corresponded to trans-TAM-4O-glucuronide and cis-TAM-4-O-glucuronide, and that peaks 5 and 7 corresponded to trans-4-OH-TAM- $N^{+}$-glucuronide and cis-4-OH-TAM- $N^{+}$-glucuronide in assays with either trans4-OH-TAM or cis-4-OH-TAM, respectively. The products corresponding to predicted trans-OH-TAM- $N^{+}$-glucuronide and cis-4-OH-TAM- $N^{+}$-glucuronide were analyzed using LC with electrospray MS detection (Figure 2). The mass spectrum for the trans-4-OH-TAM- $N^{+}$-glucuronide showed a clear $\left[\mathrm{M}^{+}\right]$ion at $\mathrm{m} / \mathrm{z} 563.80$ (calculated molecular weight, 563.29); a virtu- ally identical pattern was observed for cis-4-OH-TAM- $N^{+}$-glucuronide, with a clear $\left[\mathrm{M}^{+}\right]$ion at $\mathrm{m} / \mathrm{z} 563.70$ (data not shown). These data suggest that the glucuronides derived from both isomers were in fact monoglucuronides.

In assays with microsomes prepared from wild-type UGT1A424Pro/48Leu-overexpressing cells, significant glucuronidating activities were observed against both trans-4-OH-TAM and cis-4-OH-TAM (Figure 3). Single glucuronide peaks were observed in assays with either substrate (Figure 3a,b), with these peaks corresponding to the retention times of peaks 5 and 7 in glucuronidation assays with human liver microsomes (see Figure 1). These peaks were alkali sensitive (Figure 3c,d) and were sensitive to treatment with $\beta$-glucuronidase (results not shown), suggesting that these glucuronides corresponded to 4-OH-TAM- $N^{+}$-glucuronide. Predicted UGT1A4-induced monoglucuronides were confirmed by LC-MS for both trans4-OH-TAM and cis-4-OH-TAM (results not shown). No TAM4-O-glucuronide formation was observed in assays with either the trans or cis isomer of 4-OH-TAM with UGT1A4-overexpressing cell microsomes, and no glucuronidation activity was observed for untransfected HK293 cell homogenates for any substrate examined in this study (results not shown).

As shown in a concentration curve for both isoforms of $4-\mathrm{OH}$ TAM (Figure 4), the rate of UGT1A4-catalyzed glucuronide formation was fully saturated at approximately $10-15 \mu \mathrm{M}$ for trans-4-OH-TAM and cis-4-OH-TAM in our assay conditions. Using a concentration range of 4-OH-TAM (1-10 $\mu \mathrm{M}$ for trans-4-OH-TAM and 1-15 $\mu \mathrm{M}$ for cis-4-OH-TAM) and a specific incubation time (30 $\mathrm{min}$ ), the apparent $K_{\mathrm{m}}$ values and the $V_{\max } / K_{\mathrm{m}}$ ratios for UGT1A4-induced glucuronidation of trans- 

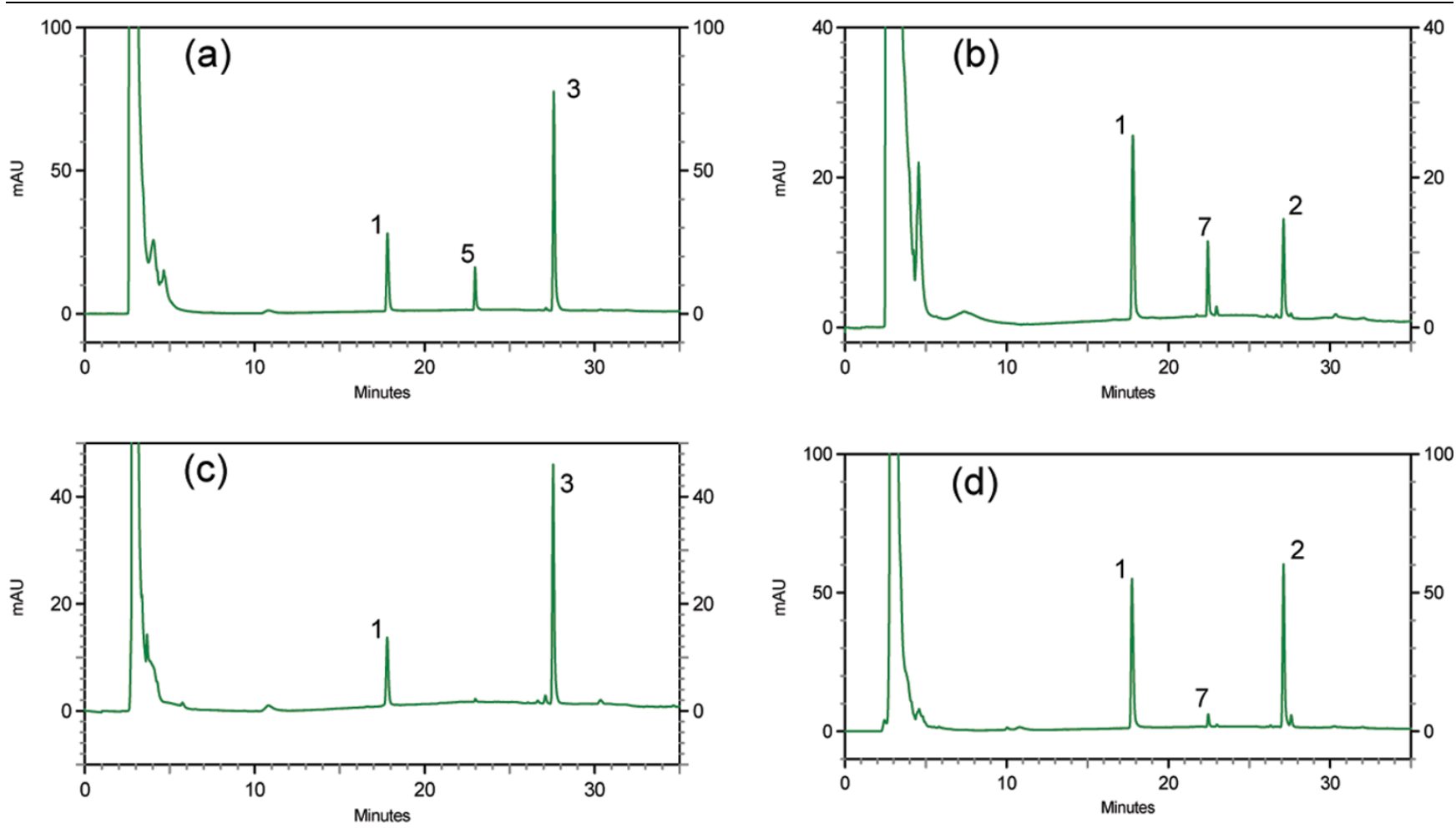

HPLC analysis of 4-hydroxytamoxifen glucuronides formed by wild-type UGT1A4-overexpressing cell microsomes. UGT1A424Pro/48Leu-overexpressing cell microsomes $\left(10 \mu \mathrm{g}\right.$ protein) were incubated at $37^{\circ} \mathrm{C}$ for $30 \mathrm{~min}$ with $25 \mu \mathrm{M}$ trans-4-hydroxytamoxifen (trans-4-OH-TAM) or $15 \mu \mathrm{M}$ cis-4-OHTAM and 4 mM UDP-glucuronic acid prior to analysis by HPLC as described in Materials and methods. Propranolol was added to all assays postincubation as an internal standard for HPLC. (a) trans-4-OH-TAM incubated with UGT1A4-overexpressing microsomes; (b) cis-4-OH-TAM incubated with UGT1A4-overexpressing microsomes; (c) trans-4-OH-TAM incubated with UGT1A4-overexpressing microsomes and $1 \mathrm{~N} \mathrm{NaOH}$; (d) cis-4$\mathrm{OH}-\mathrm{TAM}$ incubated with HLM and $1 \mathrm{~N} \mathrm{NaOH}$. Peak 1, propranolol; peak 2, cis-4-OH-TAM; peak 3, trans-4-OH-TAM; peak 5, trans-4-OH-TAM- $N^{+}-$ glucuonide; peak 7, cis-4-OH-TAM- $N^{+}$-glucuonide (peak numbers consistent with those described for Figure 1). mAU, milli-absorbance units.

4-OH-TAM and of cis-4-OH-TAM were $2.2 \pm 0.4 \mu \mathrm{M}$ and 29.3 $\pm 2.7 \mu \mathrm{l} / \mathrm{min} / \mu \mathrm{g}$, and $2.1 \pm 0.4 \mu \mathrm{M}$ and $2.0 \pm 0.3 \mu \mathrm{l} / \mathrm{min} / \mu \mathrm{g}$ respectively (performed in three independent experiments for both isomers).

\section{Analysis of TAM and trans-4-OH-TAM, cis-4-OH-TAM glucuronidation by human UGT1A4 variants}

To determine whether the Pro>Thr amino acid change at codon 24 or the Leu $>\mathrm{Val}$ amino acid change at codon 48 affected UGT1A4 enzyme activity against TAM or 4-OH-TAM isomers, stable HK293 cell lines overexpressing either the UGT1A424Tr/48Leu or the UGT1A4 ${ }^{24 P r o / 48 V a l}$ variant isoforms were created by site-directed mutagenesis using the pcDNA3.1/V5-His-TOPO plasmid expressing wild-type UGT1A424Pro/48Leu as a template. Semiquantitative western blot analysis showed high levels of UGT1A4 protein in microsomes prepared from wild-type UGT1A424Pro/48Leu-overexpressing and UGT1A424Thr/48Leu-overexpressing or UGT1A424Pro/48Val-overexpressing HK293 cell lines (Figure 5). The UGT1A4 expression levels were normalized to the levels of the endoplasmic reticulum-specific protein, calnexin, in each lane as measured by densitometry and were determined against varying amounts of human UGT1A protein (200-300 ng; also measured by densitometry). The results demonstrated that the level of expression of UGT1A4 in the UGT1A424Thr/ 48Leu-overexpressing and UGT1A424Pro/48Val-overexpressing cell lines was respectively 0.73 -fold and 0.81 -fold that observed in the wild-type UGT1A4-overexpressing cell line.

As shown in Table 1, kinetic analysis demonstrated that higher glucuronidation activities were observed for UGT1A424Pro/ $48 \mathrm{Val}$-overexpressing microsomes as compared with microsomes from wild-type UGT1A424Pro/48Leu-overexpressing cells against TAM and against both the trans and cis isomers of 4$\mathrm{OH}$-TAM. A significantly $(P \leq 0.02)$ lower $K_{\mathrm{m}}$ value $(\sim 1.6$-fold to 1.8-fold) was observed for both 4-OH-TAM isomers, while a near-significant $(P=0.053)$ decrease in $K_{\mathrm{m}}$ was observed for TAM - for the UGT1A424Pro/48Val variant compared with wild-type UGT1A4. The $V_{\max } / K_{\mathrm{m}}$ ratio for the UGT1A424Pro/ $48 \mathrm{Val}$ variant was significantly $(P \leq 0.005)$ higher than that observed for the wild-type UGT1A4 isoform after normalization for UGT1A4 expression by western blotting for both the 

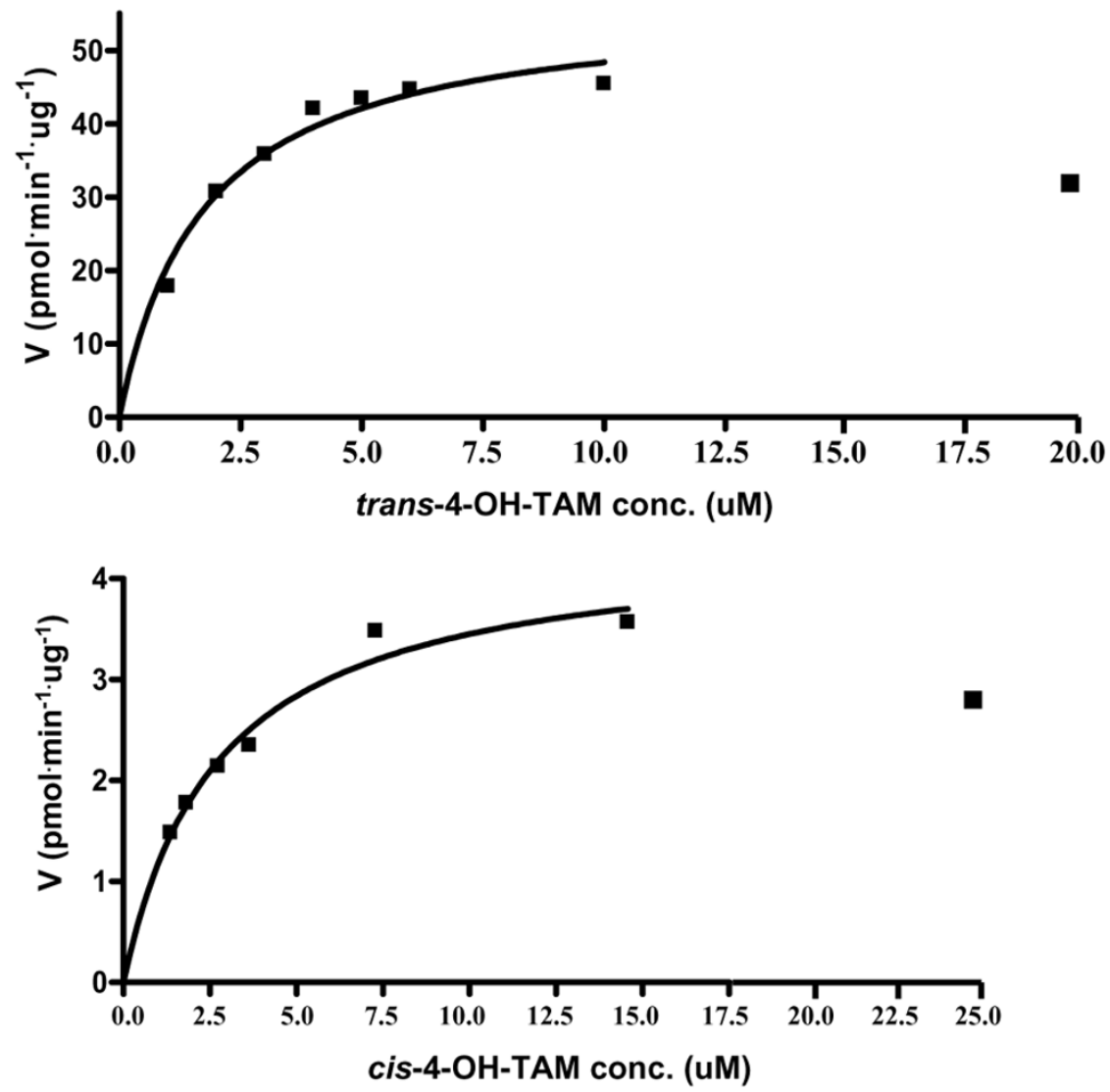

Concentration curves for 4-hydroxytamoxifen glucuronide formation with microsomes from wild-type UGT1A4-overexpressing cells. Concentration curve incubations for trans-4-hydroxytamoxifen (trans-4-OH-TAM) and cis-4-hydroxytamoxifen (cis-4-OH-TAM) glucuronide formation were performed for $30 \mathrm{~min}$ at $37^{\circ} \mathrm{C}$ using $10 \mu \mathrm{g}$ microsomal protein.

Table 1

Kinetic analysis of UGT1A4-induced glucuronidation of tamoxifen, trans-4-hydroxytamoxifen and cis-4-hydroxytamoxifen

\begin{tabular}{|c|c|c|c|c|}
\hline Substrate & UGT1A4 variant & $\begin{array}{l}V_{\max } \text { value (pmol/min } / \mu \mathrm{g} \\
\text { UGT1A4 protein) }\end{array}$ & $K_{\mathrm{m}}$ value $(\mu \mathrm{M})$ & $\begin{array}{l}V_{\text {max }} / K_{m} \text { ratio }(\mu l / m i n / \mu g \\
\text { UGT1A4 protein) }\end{array}$ \\
\hline \multirow[t]{3}{*}{ trans-4-Hydroxytamoxifen } & UGT1A424Pro/48Leu & $62.4 \pm 5.8$ & $2.2 \pm 0.4$ & $29.3 \pm 2.7$ \\
\hline & UGT1A424Thr/48Leu & $54.9 \pm 11.4$ & $1.6 \pm 0.1$ & $33.2 \pm 4.9$ \\
\hline & UGT1A424Pro/48Val & $49.3 \pm 2.8$ & $1.2 \pm 0.1^{*}$ & $40.8 \pm 1.4^{\star *}$ \\
\hline \multirow[t]{3}{*}{ cis-4-Hydroxytamoxifen } & UGT1A424Pro/48Leu & $4.1 \pm 0.1$ & $2.1+0.4$ & $2.0+0.3$ \\
\hline & UGT1A424Thr/48Leu & $5.0+0.7$ & $2.3+0.4$ & $2.2+0.5$ \\
\hline & UGT1A424Pro/48Val & $5.6+0.2$ & $1.3+0.1^{\dagger}$ & $4.4+0.4^{\ddagger}$ \\
\hline \multirow[t]{3}{*}{ Tamoxifen } & UGT1A424Pro/48Leu & $68.0 \pm 8.6$ & $2.0 \pm 0.51$ & $35.2 \pm 9.6$ \\
\hline & UGT1A424Thr/48Leu & $62.1 \pm 2.6$ & $1.5 \pm 0.20$ & $41.0 \pm 7.0$ \\
\hline & UGT1A424Pro/48Val & $52.1 \pm 10.1$ & $1.3 \pm 0.10 \S$ & $40.1 \pm 7.5$ \\
\hline
\end{tabular}

Data are expressed as the mean \pm standard deviation for three independent experiments. Values are significantly $\left({ }^{\star} P=0.01,{ }^{\star \star} P=0.005,{ }^{\dagger} P=\right.$ $\left.0.02,{ }^{\ddagger} P=0.001\right)$ or near-significantly $(\$ P=0.053)$ higher than that observed for microsomes from wild-type UGT1A424Pro/48Leu-overexpressing cells. 
Figure 5

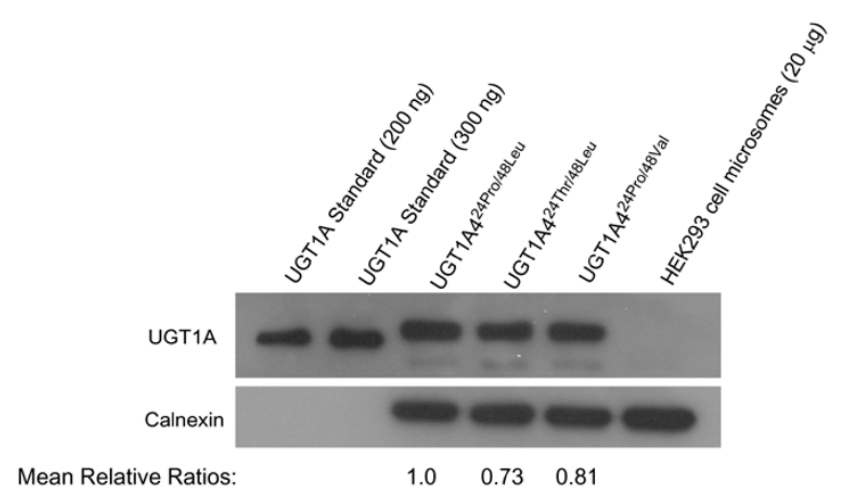

Western blot analysis of UGT1A4 protein from UGT1A4-overexpressing cell lines. Equal amounts $(20 \mu \mathrm{g})$ of microsomal protein were loaded for each lane. The amount of UGT1A4 expression was normalized against the levels of calnexin in each lane and was quantified by densitometry relative as described in Materials and methods. Untransfected HK293 cell protein was analyzed as a negative control for UDPglucuronosyltransferase detection.

enzyme kinetics was observed for the UGT1A424Thr/48Leu variant against either isomer of 4-OH-TAM or against TAM itself.

\section{Discussion}

The present study is the first to examine the potential role of UGT genetic polymorphisms on the metabolism of TAM and its active metabolites. Limited studies have previously been reported identifying the UGT enzymes involved in TAM metabolism. The hepatic UGT2B15 was shown to be the major UGT active against cis-4-OH-TAM, while a second hepatic UGT (UGT1A4) was suggested to possess limited activity against both the cis and trans isomers of 4-OH-TAM [29]. In more recent studies [30], UGT1A4 was shown to be the only active UGT against TAM, forming a quarternary ammonium-linked glucuronide with TAM's $N, N$-dimethylaminoalkyl side chain. The fact that UGT1A4 was shown to be active against TAM is not surprising given that this activity is consistent with UGT1A4's activity spectrum to produce $N$-glucuronidated metabolites with other compounds [31-34,39-41].

To better characterize the glucuronide specificity of UGT1A4 against 4-OH-TAM in the present study, HPLC assays were conducted using UGT1A4-overexpressing cells. In this study, UGT1A4 exhibited significant activity with very low apparent $K_{\mathrm{m}}$ values against both trans-4-OH-TAM and cis-4-OH-TAM. The single glucuronide peaks observed for incubations of UGT1A4-overexpressing microsomes with either trans-4-OHTAM or cis-4-OH-TAM were sensitive to alkali as well as to $\beta$ glucuronidase, suggesting that these glucuronides corresponded to 4-OH-TAM- $N^{+}$-glucuronide. This pattern of glucuronide formation was similar to that observed in human liver microsomes, where both the TAM-4-O-glucuronide and the 4$\mathrm{OH}-\mathrm{TAM}-\mathrm{N}^{+}$-glucuronide were detected in incubations with either the trans or cis isomers of 4-OH-TAM, with the peaks corresponding to 4-OH-TAM- $N^{+-}$-glucuronide sensitive to treatment with alkali and $\beta$-glucuronidase. Both trans-4-OHTAM- $N^{+}$-glucuronide and cis-4-OH-TAM- $N^{+}$-glucuronide peaks were confirmed by LC-MS to be 4-OH-TAM monoglucuronides.

These data suggest that - identical to previous observations for TAM [30], and as in recent studies by Ogura and colleagues [42] for 4-OH-TAM - UGT1A4 forms a quarternary ammonium-linked glucuronide with the $N, N$-dimethylaminoalkyl side chain of 4-OH-TAM. This activity is consistent with UGT1A4's activity spectrum to produce $N$-glucuronidated metabolites with other compounds [31-34,39-41].

The $K_{\mathrm{m}}$ values reported in the present study for wild-type UGT1A4-induced glucuronidation of trans-4-OH-TAM and cis-4-OH-TAM were $2.2 \mu \mathrm{M}$ and $2.1 \mu \mathrm{M}$, respectively. These values are 35-fold lower than that observed previously for UGT1A4 against the trans and cis isoforms of 4-OH-TAM [29]. The differences in kinetic data observed between studies for the same enzyme may be due to the fact that a baculosome overexpression system was used in the analysis of UGT1A4 in previous studies while microsomes from UGT1A4-overexpressing cells were used for the present study.

While trans-4-OH-TAM exhibits approximately 100 times the level of antiestrogenic activity compared with nonmetabolized tamoxifen and is probably a significant contributor to overall TAM-associated antiestrogenic activity [16-21], its desmethyl derivative, endoxifen, exhibits roughly the same antiestrogenic activity as 4-OH-TAM, but appears to be more abundant than 4-OH-TAM in the serum of TAM-treated women $[19,43,44]$. Studies examining the glucuronidation of endoxifen have not yet been performed, but, unlike TAM and 4-OH-TAM, the $N$ position on endoxifen is demethylated, suggesting that endoxifen may perhaps be a less effective substrate for $N$-glucuronidation by enzymes such as UGT1A4. Studies aimed to identify the UGTs that are active against endoxifen are currently ongoing.

The two previously identified missense UGT1A4 polymorphisms examined in this study are located at codons 24 and 48, resulting in Pro $>$ Thr and Leu $>V$ al amino acid changes, respectively $[35,36]$. The codon 24 polymorphism, which was previously linked to increased glucuronidation activity against the tobacco-specific nitrosamine 4(methylnitrosamino) 1(3pyridyl) 1 butanol [36] in in vitro assays using individual human liver microsomes, exhibited no association with altered glucuronidation capacity in vitro for 4-OH-TAM or for TAM in the present study. This pattern is also different from the apparent decrease in activity observed for this variant against $\beta$-naphthylamine, benzidine, trans-androsterone and dihydrotestosterone, although statistical analysis for glucuronidation rates between variants against each of these compounds were not provided in these studies [35]. This suggests that the 
functional effects of the UGT1A4 codon 24 polymorphism may be substrate dependent. Alternatively, in vitro functional analysis of the codon 24 polymorphism using a cell-line-overexpression system may not be optimal for studies of its effect on glucuronidation kinetics. A significant association was, however, observed for the UGT1A4 codon 48 polymorphism and 4-OH-TAM glucuronidation. The increased activity observed for the UGT1A424Pro/48Val variant as compared with the wild-type UGT1A424Pro/48Leu isoform against trans-4-OHTAM, cis-4-OH-TAM and TAM suggests that the codon 48 polymorphism may significantly alter UGT1A4 enzymatic function against TAM and its active metabolites. As it is not yet clear whether UGT1A4 is active against endoxifen, the role of the UGT1A424Pro/48Val variant on endoxifen glucuronidation is presently not known.

Previous studies have demonstrated that variant genotypes for enzymes involved in the TAM metabolism may significantly alter overall patient response to TAM. The low-activity SULT1A $1 * 2$ allele was linked to increased rates of mortality in breast cancer patients treated with TAM [45]. In addition, the use of antidepressants, which act to inhibit CYP2D6, resulted in significantly smaller reductions in plasma hydroxylated TAM metabolite levels in TAM-treated patients with a variant CYP2D6 genotype [19,43], supporting a role for the CYP2D6 genotype in overall patient response to TAM. Variant, lowactivity/expression alleles in CYP2D6, CYP2B6 and CYP2C9 were similarly correlated with levels of trans-4-OH-TAM formation in human liver microsomes from individual subjects [46].

The data presented in the present report. suggest that subjects with the UGT1A424Pro/48Val variant could potentially be similarly impacted with respect to individual patient response to TAM treatment and TAM-related toxicities. The prevalence of the UGT1A424Pro/48Val variant is relatively high $(9.5 \%$ in a German Caucasian population [35]) so this could impact a relatively large percentage of the population given the large number of women being treated with TAM, with approximately $19 \%$ of Caucasian females expected to have at least one UGT1A424Pro/48Val variant allele. Long-term studies examining patient response to TAM and TAM-induced toxicities versus UGT1A4 genotypes will be necessary to more fully evaluate the role of UGT genotypes on TAM therapeutic efficacy.

\section{Conclusion}

Results from this study indicate that UGT1A4 exhibits very high activity against TAM and against both isomers of $4-\mathrm{OH}$ TAM, and that the UGT1A4 codon 48 polymorphic variant is associated with altered TAM and 4-OH-TAM glucuronidation activities. These studies suggest that subjects with the UGT1A424Pro/48Val variant could potentially be similarly impacted with respect to individual patient response to TAM treatment and TAM-related toxicities.

\section{Competing interests}

The authors declare that they have no competing interests.

\section{Authors' contributions}

DXS designed the experimental plan, performed all kinetic analysis and contributed to tissue culture experiments for the studies described in this paper. GC helped to develop HPLC separation protocols and contributed to all data analysis and discussions. RWD guided all molecular aspects of this paper and contributed to mammalian tissue culture experiments and cell microsome preparations. KD performed the western blot analysis and helped to establish UGT1A4 cell lines. J-LF created the UGT1A4 cell lines. PL was instrumental in the planning, supervision and data analysis, and cowrote the manuscript with DXS. All authors participated in the revision of the manuscript and gave final approval of the version to be published.

\section{Acknowledgements}

Thanks to Arun Sharma and Telih Boyiri for their assistance with LC-MS analysis and the Penn State University College of Medicine Molecular Biology Core Facility for DNA sequencing services. These studies were supported by Public Health Service (PHS) grants R01-DE13158 (National Institute for Dental and Craniofacial Research) and P01. CA68384 (National Cancer Institute) from the National Institutes of Health, Department of Health and Human Services to PL.

\section{References}

1. Fisher B, Costantino JP, Wickerham DL, Redmond CK, Kavanah M, Cronin WM, Vogel V, Robidoux A, Dimitrov N, Atkins J, et al:: Tamoxifen for prevention of breast cancer: report of the National Surgical Adjuvant Breast and Bowel Project P-1 Study. J Natl Cancer Inst 1998, 90:1371-1388.

2. Osborne CK: Tamoxifen in the treatment of breast cancer. $N$ Engl J Med 1998, 339:1609-1618.

3. Cuzick J, Powles T, Veronesi U, Forbes J, Edwards R, Ashley S, Boyle P: Overview of the main outcomes in breast-cancer prevention trials. Lancet 2003, 361:296-300.

4. Howell A, Howell SJ, Evans DG: New approaches to the endocrine prevention and treatment of breast cancer. Cancer Chemother Pharmacol 2003, 52(Suppl 1):S39-S44.

5. Chowdhury S, Ellis P: Extended adjuvant endocrine therapy of early breast cancer. Curr Med Res Opin 2005, 21:1985-1995.

6. Nechushtan H, Peretz T: Tamoxifen and breast cancer. Harefuah 2002, 141:718-720, 761, 760.

7. McDonald CC, Stewart HJ: Fatal myocardial infarction in the Scottish adjuvant tamoxifen trial. The Scottish Breast Cancer Committee. BMJ 1991, 303:435-437.

8. Rutqvist LE, Mattsson A: Cardiac and thromboembolic morbidity among postmenopausal women with early-stage breast cancer in a randomized trial of adjuvant tamoxifen. The Stockholm Breast Cancer Study Group. J Natl Cancer Inst 1993, 85:1398-1406.

9. van Leeuwen FE, Benraadt J, Coebergh JW, Kiemeney LA, Gimbrere $\mathrm{CH}$, Otter R, Schouten LJ, Damhuis RA, Bontenbal M, Diepenhorst FW, et al:: Risk of endometrial cancer after tamoxifen treatment of breast cancer. Lancet 1994, 343:448-452.

10. Rutqvist LE, Johansson $H$, Signomklao $T$, Johansson $U$, Fornander $\mathrm{T}$, Wilking $\mathrm{N}$ : Adjuvant tamoxifen therapy for early stage breast cancer and second primary malignancies. Stockholm Breast Cancer Study Group. J Natl Cancer Inst 1995, 87:645-651.

11. Meier $\mathrm{CR}$, Jick $\mathrm{H}$ : Tamoxifen and risk of idiopathic venous thromboembolism. Br J Clin Pharmacol 1998, 45:608-612.

12. Tamoxifen for early breast cancer: an overview of therandomised trials. Early Breast Cancer Trialists' Collaborative Group. Lancet 1998, 351:1451-1467. 
13. Fisher B, Costantino JP, Redmond CK, Fisher ER, Wickerham DL, Cronin WM: Endometrial cancer in tamoxifen-treated breast cancer patients: findings from the National Surgical Adjuvant Breast and Bowel Project (NSABP) B-14. J Natl Cancer Inst 1994, 86:527-537.

14. Bernstein L, Deapen D, Cerhan JR, Schwartz SM, Liff J, McGannMaloney E, Perlman JA, Ford L: Tamoxifen therapy for breast cancer and endometrial cancer risk. J Natl Cancer Inst 1999, 91:1654-1662.

15. Bergman L, Beelen ML, Gallee MP, Hollema H, Benraadt J, van Leeuwen FE: Risk and prognosis of endometrial cancer after tamoxifen for breast cancer. Comprehensive Cancer Centres' ALERT Group. Assessment of Liver and Endometrial cancer Risk following Tamoxifen. Lancet 2000, 356:881-887.

16. Jordan VC, Collins MM, Rowsby L, Prestwich G: Amonohydroxylated metabolite of tamoxifen with potent antioestrogenic activity. J Endocrinol 1977, 75:305-316.

17. Furr BJ, Jordan VC: The pharmacology and clinical uses of tamoxifen. Pharmacol Ther 1984, 25:127-205.

18. Katzenellenbogen BS, Norman MJ, Eckert RL, Peltz SW, Mangel WF: Bioactivities, estrogen receptor interactions, and plasminogen activator-inducing activities of tamoxifen and hydroxy-tamoxifen isomers in MCF-7 human breast cancer cells. Cancer Res 1984, 44:112-119.

19. Stearns V, Johnson MD, Rae JM, Morocho A, Novielli A, Bhargava $P$, Hayes DF, Desta Z, Flockhart DA: Active tamoxifen metabolite plasma concentrations after coadministration of tamoxifen and the selective serotonin reuptake inhibitor paroxetine. $J$ Natl Cancer Inst 2003, 95:1758-1764

20. Johnson MD, Zuo H, Lee KH, Trebley JP, Rae JM, Weatherman RV, Desta Z, Flockhart DA, Skaar TC: Pharmacological characterization of 4-hydroxy-N-desmethyl tamoxifen, a novel active metabolite of tamoxifen. Breast Cancer Res Treat 2004, 85:151-159.

21. Lim YC, Desta Z, Flockhart DA, Skaar TC: Endoxifen (4-hydroxy$\mathrm{N}$-desmethyl-tamoxifen) has anti-estrogenic effects in breast cancer cells with potency similar to 4-hydroxy-tamoxifen. Cancer Chemother Pharmacol 2005, 55:471-478.

22. Buckley MM, Goa KL: Tamoxifen. A reappraisal of its pharmacodynamic and pharmacokinetic properties, and therapeutic use. Drugs 1989, 37:451-490.

23. Murphy CS, Langan-Fahey SM, McCague R, Jordan VC: Structure-function relationships of hydroxylated metabolites of tamoxifen that control the proliferation of estrogen-responsive T47D breast cancer cells in vitro. Mol Pharmacol 1990, 38:737-743.

24. Robertson DW, Katzenellenbogen JA, Long DJ, Rorke EA, Katzenellenbogen BS: Tamoxifen antiestrogens. A comparison of the activity, pharmacokinetics, and metabolic activation of the cis and trans isomers of tamoxifen. J Steroid Biochem 1982, 16:1-13.

25. Lien EA, Solheim E, Lea OA, Lundgren S, Kvinnsland S, Ueland PM: Distribution of 4-hydroxy- $N$-desmethyltamoxifen and other tamoxifen metabolites in human biological fluids during tamoxifen treatment. Cancer Res 1989, 49:2175-2183.

26. Poon GK, Chui YC, McCague R, Llnning PE, Feng R, Rowlands MG, Jarman M: Analysis of phase I and phase II metabolites of tamoxifen in breast cancer patients. Drug Metab Dispos 1993, 21:1119-1124

27. Lien EA, Solheim E, Kvinnsland S, Ueland PM: Identification of 4hydroxy- $N$-desmethyltamoxifen as a metabolite of tamoxifen in human bile. Cancer Res 1988, 48:2304-2308.

28. Nowell SA, Ahn J, Rae JM, Scheys JO, Trovato A, Sweeney C, MacLeod SL, Kadlubar FF, Ambrosone CB: Association of genetic variation in tamoxifen-metabolizing enzymes with overall survival and recurrence of disease in breast cancer patients. Breast Cancer Res Treat 2005, 91:249-258.

29. Nishiyama T, Ogura K, Nakano H, Ohnuma T, Kaku T, Hiratsuka A, Muro K, Watabe T: Reverse geometrical selectivity in glucuronidation and sulfation of cis- and trans-4-hydroxytamoxifens by human liver UDP-glucuronosyltransferases and sulfotransferases. Biochem Pharmacol 2002, 63:1817-1830.

30. Kaku T, Ogura K, Nishiyama T, Ohnuma T, Muro K, Hiratsuka A: Quaternary ammonium-linked glucuronidation of tamoxifen by human liver microsomes and UDP-glucuronosyltransferase 1A4. Biochem Pharmacol 2004, 67:2093-2102.
31. Green MD, Tephly TR: Glucuronidation of amines and hydroxylated xenobiotics and endobiotics catalyzed by expressed human UGT1.4 protein. Drug Metab Dispos 1996, 24:356-363.

32. Green MD, Tephly TR: Glucuronidation of amine substrates by purified and expressed UDP-glucuronosyltransferase proteins. Drug Metab Dispos 1998, 26:860-867.

33. Breyer-Pfaff U, Mey U, Green MD, Tephly TR: Comparative N-glucuronidation kinetics of ketotifen and amitriptyline by expressed human UDP-glucuronosyltransferases and liver microsomes. Drug Metab Dispos 2000, 28:869-872.

34. Wiener D, Doerge DR, Fang JL, Upadhyaya P, Lazarus P: Characterization of $\mathrm{N}$-glucuronidation of the lung carcinogen 4(methylnitrosamino)-1-(3-pyridyl)-1-butanol (NNAL) inhuman liver: importance of UDP-glucuronosyltransferase 1A4. Drug Metab Dispos 2004, 32:72-79.

35. Ehmer U, Vogel A, Schutte JK, Krone B, Manns MP, Strassburg CP: Variation of hepatic glucuronidation: Novel functional polymorphisms of the UDP-glucuronosyltransferase UGT1A4. Hepatology 2004, 39:970-977.

36. Wiener D, Fang JL, Dossett N, Lazarus P: Correlation between UDP-glucuronosyltransferase genotypes and 4-(methylnitrosamino)-1-(3-pyridyl)-1-butanone glucuronidation phenotype in human liver microsomes. Cancer Res 2004, 64:1190-1196.

37. Coughtrie MW, Burchell B, Bend JR: A general assay for UDPglucuronosyltransferase activity using polar amino-cyano stationary phase HPLC and UDP[U-14C]glucuronic acid. Anal Biochem 1986, 159:198-205.

38. Fang JL, Beland FA, Doerge DR, Wiener D, Guillemette C, Marques MM, Lazarus P: Characterization of benzo(a)pyrenetrans-7,8-dihydrodiol glucuronidation by human tissue microsomes and overexpressed UDP-glucuronosyltransferase enzymes. Cancer Res 2002, 62:1978-1986.

39. Kaivosaari S, Salonen JS, Taskinen J: $\mathbf{N}$-Glucuronidation of some 4-arylalkyl-1 $\mathrm{H}$-imidazoles by rat, dog, and human liver microsomes. Drug Metab Dispos 2002, 30:295-300.

40. Nakajima M, Tanaka E, Kobayashi T, Ohashi N, Kume T, Yokoi T: Imipramine $\mathrm{N}$-glucuronidation in human liver microsomes: biphasic kinetics and characterization of UDP-glucuronosyltransferase isoforms. Drug Metab Dispos 2002, 30:636-642.

41. Ren Q, Murphy SE, Dannenberg AJ, Park JY, Tephly TR, Lazarus P: Glucuronidation of the lung carcinogen 4-(methylnitrosamino)-1-(3-pyridyl)-1-butanol (NNAL) by rat UDP-glucuronosyltransferase 2B1. Drug Metab Dispos 1999, 27:1010-1016.

42. Ogura K, Ishikawa $Y$, Kaku T, Nishiyama T, Ohnuma T, Muro K, Hiratsuka A: Quaternary ammonium-linked glucuronidation of trans-4-hydroxytamoxifen, an active metabolite of tamoxifen, by human liver microsomes and UDP-glucuronosyltransferase 1A4. Biochem Pharmacol 2006, 71:1358-1369.

43. Jin Y, Desta Z, Stearns V, Ward B, Ho H, Lee KH, Skaar T, Storniolo AM, Li L, Araba A, et al:: CYP2D6 genotype, antidepressant use, and tamoxifen metabolism during adjuvant breast cancer treatment. J Natl Cancer Inst 2005, 97:30-39.

44. Desta Z, Ward BA, Soukhova NV, Flockhart DA: Comprehensive evaluation of tamoxifen sequential biotransformation by the human cytochrome P450 system in vitro: prominent roles for CYP3A and CYP2D6. J Pharmacol Exp Ther 2004, 310:1062-1075.

45. Nowell S, Sweeney C, Winters M, Stone A, Lang NP, Hutchins LF, Kadlubar FF, Ambrosone CB: Association between sulfotransferase 1A1 genotype and survival of breast cancer patients receiving tamoxifen therapy. J Natl Cancer Inst 2002, 94:1635-1640.

46. Coller JK, Krebsfaenger N, Klein K, Endrizzi K, Wolbold R, Lang T, Nussler A, Neuhaus P, Zanger UM, Eichelbaum M, et al:: The influence of CYP2B6, CYP2C9 and CYP2D6 genotypes on the formation of the potent antioestrogen Z-4-hydroxy-tamoxifen in human liver. Br J Clin Pharmacol 2002, 54:157-167. 